"C 2019 IEEE. Personal use of this material is permitted. Permission from IEEE must be obtained for all other uses, in any current or future media, including reprinting/republishing this material for advertising or promotional purposes, creating new collective works, for resale or redistribution to servers or lists, or reuse of any copyrighted component of this work in other works." 


\section{Mixed Integer Nonlinear Programming for Joint Coordination of Plug-in Electrical Vehicles Charging and Smart Grid Operations}

\author{
$1^{\text {st }}$ Y. Shi \\ School of Electrical \\ and Data Engineering, \\ University of Technology Sydney \\ Sydney, Australia \\ Ye.Shi-1@uts.edu.au
}

\author{
$2^{\text {nd }}$ H. D. Tuan \\ School of Electrical \\ and Data Engineering, \\ University of Technology Sydney \\ Sydney, Australia \\ Tuan.Hoang@uts.edu.au
}

\author{
$3^{\text {rd }}$ Andrey V. Savkin \\ School of Electrical Engineering \\ and Telecommunications, \\ The University of New South Wales \\ Sydney, Australia \\ a.savkin@unsw.edu.au
}

\begin{abstract}
The joint coordination of plug-in electric vehicles (PEVs) charging and grid power control is to minimize both PEVs' charging cost and energy generation cost in meeting both PEVs' power demands and power grid operational constraints. A bang-bang PEV charging strategy is adopted to exploit its simple online implementation, which requires computation of a mixed integer nonlinear programming problem (MINP) in binary variables of the PEV charging and continuous variables of the grid voltages. A novel solver for this challenging MINP is proposed. Its efficiency is shown by numerical simulations.

Index Terms-Smart grid, plug-in electric vehicles, bangbang charging, online algorithm, mixed integer nonlinear programming.
\end{abstract}

\section{INTRODUCTION}

In recent years, advancements in battery and smart grid technology have drawn growing attention to electric vehicles (EVs), which play an important role to mitigate the shortage of fossil fuels and severe environment pollution problems [1], [2]. It is expected that around $10 \%$ of new vehicles will be EVs by 2020 [3]. However, the massive penetration of plug-in electric vehicles (PEVs) can pose potential threats to the stability of a power grid, which is not easily compensated [4]. Unregulated charging of PEVs may cause overloading, additional power loss and unacceptable voltage violation [5]. Coordinating PEV charging is thus needed for costsaving services in meeting PEVs power demands and other consumer power demand.

To address the coordination of the PEV charging problem, [6] proposed a mixed integer nonlinear programming (MINP) model in an unbalanced system. This MINP model was then linearized to a mixed integer linear programming (MILP) model by applying the first order Taylor expansion and piecewise linear approximation. As a result, the solution of MILP is not necessarily feasible to the original MINP problem. A similar MILP model was proposed in [7] by integrating a vehicle-to-grid (V2G) charging strategy to the grid. Nevertheless, the practicability of PEVs' discharging involving costs and technologies has raised continuous concern [8]. In addition, the above references for coordinating PEV charging are all based on the off-line strategy. In that case, prior information of PEVs' charging profiles including the arrival time, departure time and state of charge $(\mathrm{SoC})$ of PEVs must be given beforehand. It is not practical to obtain all of the information in advance. To deal with the online PEV coordination, a model predictive control (MPC)-based approach has been widely used in recent studies [9], [11]. In [9], PEV charging was scheduled in a finite horizon by a MPC-based model while grid operational constraints were ignored. A MILP model over a rolling horizon window for energy storage control was developed in [10]. However, the power balance constraints in [10] were not considered, which may lead to power unbalance in the grid. Reference [11] proposed a stochastic optimization algorithm to tackle the MILP-based MPC model for various types of PEVs coordination problems, which may suffer from large computational cost.

In this paper a bang-bang strategy is adopted for PEV charging. At each time slot PEVs either charge at a maximal power rate or do not charge at all. The obvious merit of such bang-bang charging strategy is its easy and efficient online implementation. At each time slot, it requires a joint coordination of PEV charging and grid power control over a time horizon, which is a MINP with binary PEV charging variables and continuous bus voltage variables of the grid. A new two-stage approach is developed to handle this MINP. At the first stage, the MINP is equivalently reformulated to a mixed integer convex programming (MICP) and multiple matrix rank constraints. By relaxing the nonconvex rank constraints, we develop a new path-following algorithm for computation of this MICP. At the second stage, the found binary value of the PEV charging variables is then substituted into the original MINP for optimizing the bus voltage variables, for which our previously developed nonsmooth optimization algorithm [12]-[14] is ready for the solution. Simulations show that the proposed approach is capable of locating the optimal solution of this MINP.

The rest of the paper is structured as follows. Section II is devoted to an MINP-based model for the joint coordination of bang-bang PEV charging and grid power control 
with analysis on its computational challenges. Section III develops a solver for this MINP. Simulations are provided in Section IV. Section V concludes the paper.

\section{MPC FOR JOINT PEV BANG-BANG CHARGING COORDINATION AND GRID POWER CONTROL}

We consider an electric power grid with a set of buses $\mathcal{N}:=\{1,2, \ldots, N\}$ connected through a set of flow lines $\mathcal{L} \subseteq \mathcal{N} \times \mathcal{N}$, under which bus $k$ is connected to bus $m$ if and only if $(k, m) \in \mathcal{L}$. Denote by $\mathcal{N}(k)$ the set of other buses connected to bus $k . \mathcal{G} \subseteq \mathcal{N}$ is the set of those buses that are connected to distributed generators (DGs). Bus $k \in$ $\mathcal{N} \backslash \mathcal{G}$ is not connected to DGs and bus $k \in \mathcal{G}$ also has a function to serve PEVs and will be referred as charging station (CS) $k$. Thus, there are $M=|\mathcal{G}|$ CSs in the grid. The serving time period of the grid is divided into $T$ time slots $\mathcal{T}:=\{1,2, \ldots, T\}$.

Denote by $\mathcal{H}_{k}$ the set of those PEVs that arrive at CS $k$. Accordingly, $k_{n}$ is the $n$-th PEV that arrives at CS $k$. Each PEV $k_{n}$ arrives at $t_{a, k_{n}} \in \mathcal{T}$ and requires to be fully charged by its departing time $t_{k_{n}, d} \in \mathcal{T}$. Suppose that $C_{k_{n}}$ and $s_{k_{n}}^{0}$ are the battery capacity and initial SOC of PEV $k_{n}$ while $P_{k_{n}}$ is the maximum power that its battery can charge during one time slot. In this paper, we adopt the bang-bang charging strategy, under which PEV $k_{n}$ either charges the maximal power $\bar{P}_{k_{n}}$ or does not charge at all at each time slot. We use the binary variable

$$
\tau_{k_{n}}\left(t^{\prime}\right) \in\{0,1\}
$$

to implement this strategy, i.e. PEV $k_{n}$ charges the power $P_{k_{n}}\left(t^{\prime}\right)=\tau_{k_{n}}\left(t^{\prime}\right) \bar{P}_{k_{n}}$ during the time slot $t^{\prime}$. Accordingly, the following constraint enables PEV $k_{n}$ to be fully charged at its departure:

$$
\sum_{t^{\prime}=t_{k_{n}, a}}^{t_{k_{n}, d}} \tau_{k_{n}}\left(t^{\prime}\right)=\bar{\tau}_{k_{n}},
$$

where $u_{h}$ is the charging efficiency of the battery, $\bar{\tau}_{k_{n}}:=$ $\left\lceil\frac{C_{k_{n}}\left(1-s_{k_{n}}^{0}\right)}{u_{h} \bar{P}_{k_{n}}}\right\rceil$. For ease of presentation, we set $\tau_{k_{n}}\left(t^{\prime}\right)=0$ for $t^{\prime} \notin\left[t_{k_{n}, a}, t_{k_{n}, b}\right]$.

From the grid side, let $y_{k m} \in \mathbb{C}$ be the admittance of line $(k, m)$ and $V_{k}\left(t^{\prime}\right)$ represent the complex voltage at bus $k$ during the time slot $t^{\prime}$. The total supply and demand energy is balanced as:

$$
\begin{array}{r}
V_{k}\left(t^{\prime}\right)\left[\sum_{m \in \mathcal{N}(k)} y_{k m}\left(V_{k}-V_{m}\right)\right]^{*}=\left[P_{g_{k}}\left(t^{\prime}\right)-P_{l_{k}}\left(t^{\prime}\right)\right. \\
\left.-\sum_{n \in \mathcal{H}_{k}} \bar{P}_{k_{n}} \tau_{k_{n}}\left(t^{\prime}\right)\right]+j\left[Q_{g_{k}}\left(t^{\prime}\right)-Q_{l_{k}}\left(t^{\prime}\right)\right], k \in \mathcal{G}, \\
V_{k}(t)\left[\sum_{m \in \mathcal{N}(k)} y_{k m}\left(V_{k}-V_{m}\right)\right]^{*}= \\
-P_{l_{k}}\left(t^{\prime}\right)-j Q_{l_{k}}\left(t^{\prime}\right), \quad k \in \mathcal{N} \backslash \mathcal{G},
\end{array}
$$

where $P_{l_{k}}\left(t^{\prime}\right)$ and $Q_{l_{k}}\left(t^{\prime}\right)$ are respectively known real and reactive price-inelastic demands to express the residential power demand, $P_{g_{k}}\left(t^{\prime}\right)$ and $Q_{g_{k}}\left(t^{\prime}\right)$ are the real and reactive powers generated by DG $k$.
The following standard constraints are also set.

- The range of generated powers by the DGs:

$$
\begin{aligned}
& \underline{P}_{g_{k}} \leq P_{g_{k}}\left(t^{\prime}\right) \leq \bar{P}_{g_{k}}, \quad k \in \mathcal{G}, \\
& \underline{Q}_{g_{k}} \leq Q_{g_{k}}\left(t^{\prime}\right) \leq \bar{Q}_{g_{k}}, \quad k \in \mathcal{G},
\end{aligned}
$$

where $\underline{P}_{g_{k}}, \underline{Q}_{g_{k}}$ and $\bar{P}_{g_{k}}, \bar{Q}_{g_{k}}$ are respectively lower and upper physical limits of the real generated and reactive generated powers.

- Voltage range and phase balance:

$$
\begin{array}{r}
\underline{V}_{k} \leq\left|V_{k}\left(t^{\prime}\right)\right| \leq \bar{V}_{k}, \\
\left|\arg \left(V_{k}\left(t^{\prime}\right)\right)-\arg \left(V_{m}\left(t^{\prime}\right)\right)\right| \leq \theta_{k m}^{\max }, \\
k \in \mathcal{N},(k, m) \in \mathcal{L}, t^{\prime} \in \mathcal{T},
\end{array}
$$

where $\underline{V}_{k}$ and $\bar{V}_{k}$ are the lower limit and upper limit of the voltage amplitude, while $\theta_{k, m}^{\max }$ are given to express the voltage phase balance.

The cost function is defined as the sum of the energy cost to DGs and charging cost for PEVs

$$
\mathcal{F}(\mathcal{R}, \tau)=\sum_{t^{\prime} \in \mathcal{T}} \sum_{k \in \mathcal{G}} f\left(P_{g_{k}}\left(t^{\prime}\right)\right)+\sum_{t^{\prime} \in \mathcal{T}} \sum_{k \in \mathcal{N}} \sum_{n \in \mathcal{H}_{k}} \beta_{t} \tau_{k_{n}}\left(t^{\prime}\right) \bar{P}_{k_{n}},
$$

where $f\left(P_{g_{k}}\left(t^{\prime}\right)\right)$ is the cost function of real power generation by DGs, which is linear or quadratic in $P_{g_{k}}\left(t^{\prime}\right)$, and $\beta_{t}$ is the known PEV charging price during the time slot $t^{\prime}$.

By defining

$$
R\left(t^{\prime}\right)=\left\{P_{g}\left(t^{\prime}\right), Q_{g}\left(t^{\prime}\right)\right\}, \mathcal{R}=\left\{R\left(t^{\prime}\right)\right\}_{t^{\prime} \in \mathcal{T}},
$$

and

$$
\tau=\left\{\tau\left(t^{\prime}\right)\right\}_{t^{\prime} \in \mathcal{T}}, \tau\left(t^{\prime}\right)=\left\{\tau_{k_{n}}\left(t^{\prime}\right)\right\}_{k_{n} \in \mathcal{H}_{k}},
$$

$\left(R\left(t^{\prime}\right), \tau\left(t^{\prime}\right)\right)$ and $V\left(t^{\prime}\right)$ are considered as the system state and control, respectively. As such, the joint PEV charging coordination and voltage control to optimize the energy and charging costs appears to be the following control problem over the finite horizon $[1, T]$ :

$$
\min _{\mathcal{V}, \mathcal{R}, \tau} \mathcal{F}(\mathcal{R}, \tau) \quad \text { s.t. } \quad(1),(2),(3)-(8) .
$$

However, all equations in (10) are not known a priori.

Denote by $C(t)$ the set of PEVs that need to be charged at $t$ and ahead. For each $k_{n} \in C(t)$, let $d_{k_{n}}(t)$ be its remaining demand for charging by the departure time $t_{k_{n}, d}$. Therefore, the binary variable

$$
\tau_{k_{n}}\left(t^{\prime}\right) \in\{0,1\}, t^{\prime} \in\left[t, t_{k_{n}, d}\right], k_{n} \in C(t)
$$

must satisfy the following constraints:

$$
\sum_{t^{\prime}=t}^{t_{k_{n}, b}} \tau_{k_{n}}\left(t^{\prime}\right)=\bar{\tau}_{k_{n}}(t), \quad k_{n} \in C(t),
$$

where $\bar{\tau}_{k_{n}}(t):=\left\lceil\frac{d_{k_{n}}(t)}{u_{h} \bar{P}_{k_{n}}}\right\rceil$. Define $\Psi(t)=\max _{k_{n} \in C(t)} t_{k_{n}, d}$, we propose an online algorithm, which at time $t$ solves the following MPC over the prediction horizon $[t, \Psi(t)]$ but then takes only $V(t), R(t)$ and $\tau(t)$, for updating the solution of (10):

$$
\min _{\mathcal{V}_{P}(t), \mathcal{R}_{P}(t), \tau_{P}(t)} F_{P}\left(\mathcal{R}_{P}(t), \tau_{P}(t)\right)
$$




$$
\begin{array}{r}
\text { s.t. } \quad(4)-(8),(11),(12), \\
V_{k}\left(t^{\prime}\right)\left[\sum_{m \in \mathcal{N}(k)} y_{k m}\left(V_{k}\left(t^{\prime}\right)-V_{m}\left(t^{\prime}\right)\right)\right]^{*} \\
=\left[P_{g_{k}}\left(t^{\prime}\right)-P_{l_{k}}\left(t^{\prime}\right)-\sum_{k_{n} \in C(t)} \bar{P}_{k_{n}} \tau_{k_{n}}\left(t^{\prime}\right)\right]+ \\
j\left(Q_{g_{k}}\left(t^{\prime}\right)-Q_{l_{k}}\left(t^{\prime}\right)\right), \quad\left(t^{\prime}, k\right) \in[t, \Psi(t)] \times \mathcal{G} .
\end{array}
$$

One can see (13) is a difficult MINP because (4), (8) and (13b) are nonlinear in the voltage variable $V\left(t^{\prime}\right)$ while (11) is a discrete combinatoric constraint. In the next section, we propose an efficient approach, which also exploits the fact that only the snapshot at $t$ of the solution of (13) is extracted to update the online solution of (10).

\section{SOLVER FOR MINP}

For $W\left(t^{\prime}\right):=V\left(t^{\prime}\right) V^{H}\left(t^{\prime}\right) \in \mathbb{C}^{N \times N}$, which must satisfy $W\left(t^{\prime}\right) \succeq 0$ and $\operatorname{rank}\left(W\left(t^{\prime}\right)\right)=1$, we replace $W_{k m}\left(t^{\prime}\right)=$ $V_{k}\left(t^{\prime}\right) V_{m}^{*}\left(t^{\prime}\right),(k, m) \in \mathcal{N} \times \mathcal{N}$ in (13) to reformulate it to the following MINP in matrix-valued variable $\mathcal{W}_{P}(t):=$ $\left\{W\left(t^{\prime}\right)\right\}_{t^{\prime} \in[t, \Psi(t)]}$ and binary-valued variable $\tau_{P}(t)$ :

$$
\begin{array}{r}
\min _{\mathcal{W}_{P}(t), \mathcal{R}_{P}(t), \tau_{P}(t)} F_{P}\left(\mathcal{R}_{P}(t), \tau_{P}(t)\right) \quad \text { s.t. }(6),(11),(12), \\
\sum_{m \in \mathcal{N}(k)}\left(W_{k k}\left(t^{\prime}\right)-W_{k m}\left(t^{\prime}\right)\right) y_{k m}^{*}=\left[P_{g_{k}}\left(t^{\prime}\right)-P_{l_{k}}\left(t^{\prime}\right)\right. \\
\left.-\sum_{k_{n} \in C(t)} \bar{P}_{k_{n}} \tau_{k_{n}}\left(t^{\prime}\right)\right]+j\left(Q_{g_{k}}\left(t^{\prime}\right)-Q_{l_{k}}\left(t^{\prime}\right)\right), \quad k \in \mathcal{G},(14 \mathrm{a}) \\
\sum_{m \in \mathcal{N}(k)}\left(W_{k k}\left(t^{\prime}\right)-W_{k m}\left(t^{\prime}\right)\right) y_{k m}^{*}= \\
-P_{l_{k}}\left(t^{\prime}\right)-j Q_{l_{k}}\left(t^{\prime}\right), k \in \mathcal{N} \backslash \mathcal{G},(14 \mathrm{~b}) \\
\underline{V}_{k}^{2} \leq W_{k k}\left(t^{\prime}\right) \leq \bar{V}_{k}^{2}, \quad k \in \mathcal{N},(14 \mathrm{c}) \\
\Im\left(W_{k m}\left(t^{\prime}\right)\right) \leq \Re\left(W_{k m}\left(t^{\prime}\right)\right) \tan \left(\theta_{k m}^{\max }\right),(k, m) \\
W\left(t^{\prime}\right) \succeq 0,(14 \mathrm{~d}) \\
\operatorname{rank}\left(W\left(t^{\prime}\right)\right)=1,(14 \mathrm{f})
\end{array}
$$

The difficulty of (14) is concentrated on the multiple nonconvex matrix rank-one constraints in (14f) and multiple binary constraints in (11). Below we propose a two-stage optimization approach to its online algorithm. In the first optimization stage, we drop the matrix rank-one constraint (14f) to relax (14) to the following MICP for $t^{\prime} \in[t, \Psi(t)]$ :

$$
\begin{array}{r}
\min _{\mathcal{W}_{P}(t), \mathcal{R}_{P}(t), \tau_{P}(t)} F_{P}\left(\mathcal{R}_{P}(t), \tau_{P}(t)\right) \\
\text { s.t. }(6),(11),(12),(14 a)-(14 e) .
\end{array}
$$

Suppose that $\left(\hat{\mathcal{W}}_{P}(t), \hat{\mathcal{R}}_{P}(t)\right), \hat{\tau}_{P}(t)$ is the optimal solution of (15). If $\operatorname{rank}\left(\hat{W}\left(t^{\prime}\right)\right) \equiv 1, t^{\prime} \in[t, \Psi(t)]$, then $\hat{V}\left(t^{\prime}\right)$ such that $\hat{W}\left(t^{\prime}\right)=\hat{V}\left(t^{\prime}\right) \hat{V}^{H}\left(t^{\prime}\right)$ together with $\hat{R}\left(t^{\prime}\right)$ and $\hat{\tau}_{k_{n}}\left(t^{\prime}\right)$ constitute the optimal solution of MINP (13). Otherwise, we go to next optimization stage, which substitutes $\hat{\tau}_{k_{n}}(t)$ into (14a) and considers the snapshot at $t$ of (14) only

$$
\begin{aligned}
&\left.\min _{W(t), R(t)} F\left(P_{g}(t)\right)\right):=\sum_{t^{\prime}=t}^{\Psi(t)} \sum_{k \in \mathcal{G}} f\left(P_{g_{k}}\left(t^{\prime}\right)\right) \\
& \text { s.t. } \quad(6),(14 b)-(14 e)(16 a)
\end{aligned}
$$

$$
\begin{array}{r}
\sum_{m \in \mathcal{N}(k)}\left(W_{k k}(t)-W_{k m}(t)\right) y_{k m}^{*}=\left[P_{g_{k}}(t)-P_{l_{k}}(t)\right. \\
\left.-\sum_{k_{n} \in C(t)} \bar{P}_{k_{n}} \hat{\tau}_{k_{n}}(t)\right]+j\left(Q_{g_{k}}(t)-Q_{l_{k}}(t)\right), \quad k \in \mathcal{G},(16 \mathrm{~b}) \\
\operatorname{rank}(W(t))=1,(16 \mathrm{c})
\end{array}
$$

which involves only one matrix rank-one constraint (16c). The rationale behind this simplified treatment is that in the end we need only the snapshot at $t$ of the solution of (14) for online updating the voltage $V(t)$ and generated power $R(t)$.

In the next two subsections we propose algorithms for solving MICP (15) and the nonconvex optimization problem (16).

\section{A. New computational solution for MICP problem (15)}

It is clear that the main task is how to cope with the discrete constraint (11) in MICP (15). The following result establishes the equivalence between this discrete constraint and a set of continuous constraints.

Lemma 1: Under the linear constraint (12), the binary constraint (11) is equivalent to the following set of continuous constraints:

$$
\begin{array}{r}
0 \leq \tau_{k_{n}}\left(t^{\prime}\right) \leq 1, t^{\prime} \in\left[t, t_{k_{n}, d}\right], k_{n} \in C(t), \\
g\left(\tau_{P}(t)\right) \geq \bar{\tau}(t):=\sum_{k_{n} \in C(t)} \bar{\tau}_{k_{n}}(t),
\end{array}
$$

where

$$
g\left(\tau_{P}(t)\right):=\sum_{k_{n} \in C(t)} \sum_{t^{\prime}=t} t_{k_{n}, b} \tau_{k_{n}}^{L}\left(t^{\prime}\right),
$$

and $\tau_{k_{n}}^{L}\left(t^{\prime}\right)$ is $\tau_{k_{n}}\left(t^{\prime}\right)$ to the power of $L>1$.

Proof. Note that

$$
\begin{aligned}
\tau_{k_{n}}^{L}\left(t^{\prime}\right) & \leq \tau_{k_{n}}\left(t^{\prime}\right) \quad \forall \tau_{k_{n}}\left(t^{\prime}\right) \in[0,1], \\
g\left(\tau_{P}(t)\right) & \leq \sum_{k_{n} \in C(t)} \sum_{t^{\prime}=t}^{t_{k_{n}, d}} \tau_{k_{n}}\left(t^{\prime}\right)=\bar{\tau}(t) .
\end{aligned}
$$

Therefore constraint (18) forces $g\left(\tau_{P}(t)\right)=\bar{\tau}(t)$, which is possible if and only if $\tau_{k_{n}}^{L}\left(t^{\prime}\right)=\tau_{k_{n}}\left(t^{\prime}\right)$, i.e. $\tau_{k_{n}}\left(t^{\prime}\right) \in$ $\{0,1\}$, implying (11).

Since $g\left(\tau_{P}(t)\right)$ is convex in $\tau_{P}(t)$, constraint (18) is called reverse convex [16]. Also, as $L$ decreases, $g\left(\tau_{P}(t)\right)$ approaches the linear function $\sum_{k_{n} \in C(t)} \sum_{t^{\prime}=t}^{t_{k_{n}, b}} \tau_{k_{n}}\left(t^{\prime}\right)$ and as thus the constraint (18) approaches the linear constraint $\sum_{k_{n} \in C(t)} \sum_{t^{\prime}=t}^{t_{n}, b} \tau_{k_{n}}\left(t^{\prime}\right) \geq \bar{\tau}(t)$. However, it does not mean that choosing $L$ closer to 1 is effective because the function $g\left(\tau_{P}(t)\right)-\bar{\tau}(t)$ also approaches zero very quickly, making constraint (18) highly artificial. In our previous works [17], [18], $L=2$ was chosen. However, we choose $L=1.5$ in this paper as it accelerates the convergence of the proposed optimization algorithms.

The following result is a direct consequence of Lemma 1.

Proposition 1: Under the linear constraint (12), the function

$$
g_{1}\left(\tau_{P}(t)\right):=\frac{1}{g\left(\tau_{P}(t)\right)}-\frac{1}{\bar{\tau}(t)}
$$


can be used to measure the degree of satisfaction of the binary constraint (11) in the sense that $g_{1}\left(\tau_{P}(t)\right) \geq$ $0 \forall \tau_{k_{n}}\left(t^{\prime}\right) \in[0,1]$ and $g_{1}\left(\tau_{P}(t)\right)=0$ if and only if $\tau_{k_{n}}\left(t^{\prime}\right)$ are binary (i.e. satisfying (11)).

Therefore MICP (15) is equivalent to the following penalized optimization problem:

$$
\begin{array}{r}
\min _{\mathcal{W}_{P}(t), \mathcal{R}_{P}(t), \tau_{P}(t)} \Phi\left(\mathcal{R}_{P}(t), \tau_{P}(t)\right):= \\
F_{P}\left(\mathcal{R}_{P}(t), \tau_{P}(t)\right)+\mu g_{1}\left(\tau_{P}(t)\right)
\end{array}
$$

s.t. $\quad(6)$ for $t^{\prime} \in[t, \Psi(t)],(12),(14 a)-(14 e),(17)$,

where $\mu>0$ is a penalty parameter. As the function $g\left(\tau_{P}(t)\right)$ is convex, it is true that at $\tau_{P}^{(\kappa)}(t)$ [16],

$$
\begin{aligned}
g\left(\tau_{P}(t)\right) \geq & g^{(\kappa)}\left(\tau_{P}(t)\right) \\
:= & g\left(\tau_{P}^{(\kappa)}(t)\right)+\left\langle\nabla g\left(\tau_{P}^{(\kappa)}(t), \tau_{P}(t)-\tau_{P}^{(\kappa)}(t)\right\rangle\right. \\
= & -(L-1) \sum_{k_{n} \in C(t)} \sum_{t^{\prime}=t}^{t_{k_{n}, d}}\left(\tau_{k_{n}}^{(\kappa)}\left(t^{\prime}\right)\right)^{L} \\
& +L \sum_{k_{n} \in C(t)} \sum_{t^{\prime}=t}^{t_{k_{n}, d}}\left(\tau_{k_{n}}^{(\kappa)}\left(t^{\prime}\right)\right)^{L-1} \tau_{k_{n}}\left(t^{\prime}\right) .
\end{aligned}
$$

Therefore, an upper bounding approximation at $\tau_{P}^{(\kappa)}(t)$ for $g_{1}\left(\tau_{P}(t)\right)$ can be easily obtained as

$$
g_{1}\left(\tau_{P}(t)\right) \leq g_{1}^{(\kappa)}\left(\tau_{P}(t)\right):=\frac{1}{g^{(\kappa)}\left(\tau_{P}(t)\right)}-\frac{1}{\bar{\tau}(t)}
$$

over the trust region

$$
g^{(\kappa)}\left(\tau_{P}(t)\right)>0
$$

Accordingly, at the $\kappa$-th iteration we solve the following convex optimization problem to generate the next iterative point $\left(\mathcal{W}_{P}^{(\kappa+1)}(t), \mathcal{R}_{P}^{(\kappa+1)}(t), \tau_{P}^{(\kappa+1)}(t)\right)$ :

$$
\begin{array}{r}
\min _{\mathcal{W}_{P}(t), \mathcal{R}_{P}(t), \tau_{P}(t)} \Phi^{(\kappa)}\left(\mathcal{R}_{P}(t), \tau_{P}(t)\right):= \\
F_{P}\left(\mathcal{R}_{P}(t), \tau_{P}(t)\right)+\mu g_{1}^{(\kappa)}\left(\tau_{P}(t)\right) \quad \text { s.t. } \quad(6), \\
\text { for } t^{\prime} \in[t, \Psi(t)],(12),(14 a)-(14 e),(17),(22) .
\end{array}
$$

We thus arrive at

$$
\begin{aligned}
& \Phi\left(\mathcal{R}_{P}^{(\kappa+1)}(t), \tau_{P}^{(\kappa+1)}(t)\right) \leq \\
& \Phi^{(\kappa)}\left(\mathcal{R}_{P}^{(\kappa+1)}(t), \tau_{P}^{(\kappa+1)}(t)\right)< \\
& F\left(\mathcal{R}_{P}^{(\kappa)}(t), \tau_{P}^{(\kappa)}(t)\right),
\end{aligned}
$$

implying that $\tau_{P}^{(\kappa+1)}(t)$ is a better feasible point than $\tau_{P}^{(\kappa)}(t)$ for (19). For a sufficiently large $\mu>0, g\left(\tau_{P}^{(\kappa)}(t)\right) \rightarrow 0$ as well, yielding an optimal solution of MICP (15). Pseudocode for this computational procedure is provided by Algorithm 1 .

\section{B. Computational procedure for (16)}

Following our previous works [12], [13], [15], [19], [20], the matrix rank-one constrained optimization problem (16)

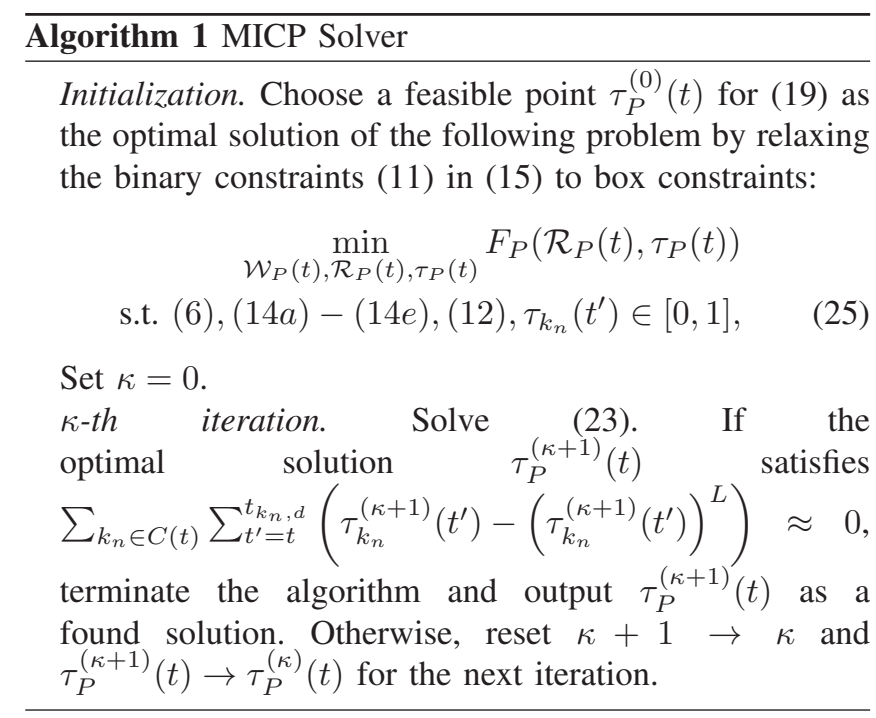

is solved via the following penalized optimization problem for $\lambda>0$ :

$\min _{W(t), R(t)} F\left(P_{g}(t)\right)+\lambda\left(\operatorname{Trace}(W(t))-\lambda_{\max }(W(t))\right),(26 \mathrm{a})$
s.t. $\quad(6),(14 b)-(14 e)$ for $t^{\prime}=t,(26 \mathrm{~b})$

which is computed by solving the following convex optimization problem at the $\kappa$ th iteration to generate $W^{(\kappa+1)}(t)$ :

$$
\begin{array}{r}
\min _{W(t), R(t)} F\left(P_{g}(t)\right)+\lambda(\operatorname{Trace}(W(t)) \\
\left.-\left(w_{\max }^{(\kappa)}(t)\right)^{H} W(t) w_{\max }^{(\kappa)}(t)\right) \text { s.t. }(26 b),
\end{array}
$$

where $W^{(k)}(t)$ is a point found from the $(\kappa-1)$ th iteration and $w_{\max }^{(\kappa)}(t)$ denotes the normalized eigenvector corresponding to the maximal eigenvalue $\lambda_{\max }\left(W^{(\kappa)}(t)\right)$ of $W^{(\kappa)}(t)$. The rationale behind using the penalized optimization problem (26) is that Trace $(W(t))-\lambda_{\max }(W(t))$ is the degree of satisfaction of the matrix rank-one constraint (16c). The reader is referred to [13] for proof of its convergence.

\section{Simulation Results}

Sedumi [21] interfaced by CVX [22] on a Core i7-7600U processor is used to solve convex optimization problems such as (23) and (27). The tolerance $\epsilon=10^{-3}$ is set for the stop criteria in the optimization algorithms. The simulated power grids are Case9, Case14, Case30 and Case57 from Matpower [23] with structure, physical limits and cost functions $f\left(P_{g_{k}}\left(t^{\prime}\right)\right)$ given in the Matpower library [23]. Table I contains their main parameters, where the second column provides the numbers of buses, generators and branches, the third column provides the dimension of $W(t)$ and the last column provides the total number of PEVs.

The considered charging period is from 6:00 pm to 6:00 am of the next day to reflect the fact that most PEVs are charged after their owners' working hours. This time period is uniformly divided into 24 30-minute time slots. The PEVs arrive during the time period from 6:00 pm to midnight. The arrival times of PEVs are independent and are generated by a truncated normal distribution $\left(20,1.5^{2}\right)$. The battery 
TABLE I

INFORMATION ON FOUR NETWORKS

\begin{tabular}{cccc}
\hline & Buses/Generators/Branches & Dim. of $W(t)$ & PEVs \\
\hline Case9 & $9 / 3 / 9$ & $\mathbb{C}^{9 \times 9}$ & 126 \\
Case14 & $14 / 5 / 20$ & $\mathbb{C}^{14 \times 14}$ & 210 \\
Case30 & $30 / 6 / 24$ & $\mathbb{C}^{30 \times 30}$ & 252 \\
Case57 & $57 / 7 / 80$ & $\mathbb{C}^{57 \times 57}$ & 294 \\
\hline
\end{tabular}

capacity $C_{k_{n}}=100 \mathrm{KWh}$ of PEVs is that of Tesla Model $\mathrm{S}$. The initial SOC $s_{k_{n}}^{0}$ of all PEVs is set as $20 \%$. The maximal charging power $u_{h} \bar{P}_{k_{n}}$ per time slot is set to be equivalent to $10 \%$ of the battery capacity, i.e. every PEV needs to charge $\bar{P}_{k_{n}}$ in 8 time slots. We set $t_{k_{n}, d}-t_{k_{n}, a} \equiv$ 12 (PEVs are required to be fully charged 6 hours after their arrivals). The price-inelastic load $P_{l_{k}}(t)$ is defined as $P_{l_{k}}(t)=l(t) \times \bar{P}_{l_{k}} \times T / \sum_{t=1}^{24} l(t)$, where $\bar{P}_{l_{k}}$ is the load demand specified by [23] and $l(t)$ is the residential load demand taken from the UK [24]. The time varying energy price is taken from [25] on different days in 2017. The data for profile I is from 6:00 pm on May 17th to 6:00 am on May 18th, for Profile 2 is from 6:00 pm on May 18th to 6:00 am on May 19th, for Profile 3 is from 6:00 pm on May 19th to 6:00 am on May 20th, and for Profile 4 is from 6:00 pm on May 15th to 6:00 am on May 16th.

The computational results are summarized in Table II. Its third column provides the number of binary variables $\tau_{k_{n}}\left(t^{\prime}\right)$ in (10). The values of the penalty parameters $\mu$ in (23) and $\lambda$ in (27) are specified in the forth and fifth columns. The value of the cost objective (9) is calculated by summing up $T$ values $\sum_{k \in \mathcal{G}} f\left(P_{g_{k}}(t)\right)+\sum_{k \in \mathcal{N}} \sum_{n \in \mathcal{H}_{k}} \beta_{t} \tau_{k_{n}}(t) \bar{P}_{k_{n}}$ with solution $\tau_{k_{n}}(t)$ and $R(t)$ of (15) and (16) and is given by the sixth and seventh columns, respectively. The effectiveness of using (16) is confirmed by observing that these values are almost the same. The average running time of computation at each time slot is shown in the last column.

Fig. 1 provides the number of iterations that Algorithm 1 needs for solving the penalized optimization problem (19) for each time $t$. Recall that at each iteration, the convex optimization problem (23) is solved, so this number of iterations is also the number of convex optimization problems (23) used in computing (19). One can see that Algorithm 1 converges rapidly within five iterations. Fig. 2 plots the aggregated charging rate for the four networks under the four residential profiles. These aggregated rates increase gradually until the midnight and then drop continuously. Their peak value is attained at 11:30 pm or 0:00 am after the integration of all PEVs into the grid. The charging rates are relatively low from $8 \mathrm{pm}$ to $10 \mathrm{pm}$ to avoid the peak of the electricity price. Fig.3 plot the SoC of four PEVs randomly taken from four cases, which arrive at different times. For a few time slots, PEVs do not charge so their SoC remain unchanged.

\section{CONCLUSions}

The joint online coordination of PEV bang-bang charging and power control to serve both PEVs and residential power
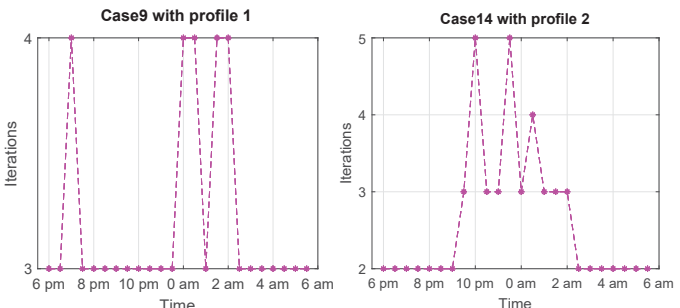

Case 30 with profile 3
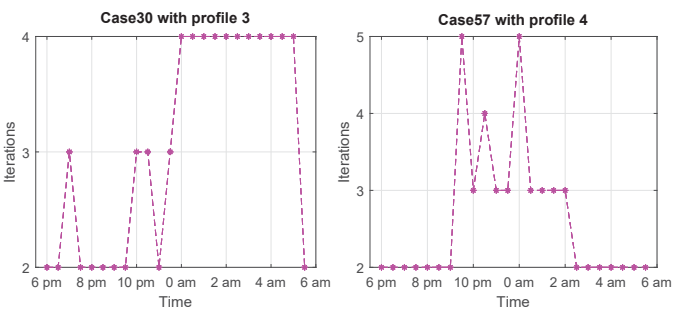

Fig. 1. Convergence performance of Algorithm 1
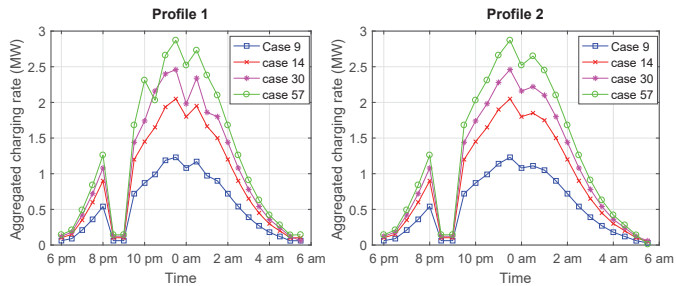

Profile 3
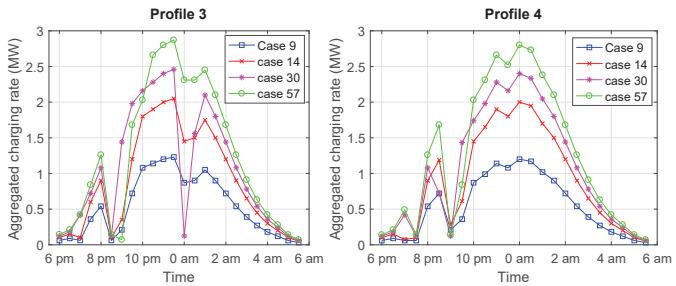

Fig. 2. Aggregated charging rate for four networks during the charging period

demands at competitive operating cost is very difficult due to the random nature of PEVs' arrivals and demands and the discrete nature of bang-bang charging. We have proposed a novel and easily-implemented MPC-based two-optimization stage online algorithm that can achieve an optimal solution.

\section{REFERENCES}

[1] Edison Electric Institute, Transportation electrification: Utility fleets leading the charge (white paper), EEI, 2014.

[2] Zhang, Tian and Chen, Wei and Han, Zhu and Cao, Zhigang, Charging scheduling of electric vehicles with local renewable energy under uncertain electric vehicle arrival and grid power price, IEEE Trans. Vehicular Technology, vol. 63, no. 6, pp. 2600-2612, 2014.

[3] Scrosati, Bruno and Garche, Jürgen and Tillmetz, Werner, Advances in battery technologies for electric vehicles, Woodhead Publishing, 2015.

[4] S. Lakshminarayana, Y. Xu, H. V. Poor, T. Q. S. Quek, Cooperation of storage operation in a power network with renewable generation, IEEE Trans. Smart Grid, vol. 7, no. 4, pp. 2108-2122, 2016.

[5] S. Huang, H. Safiullah, J. Xiao, B.-M. S. Hodge, R. Hoffman, J. Soller, D. Jones, D. Dininger, W. E. Tyner, A. Liu, et al., The effects of electric vehicles on residential households in the city of indianapolis, Energy Policy, vol. 49, pp. 442-455, 2012. 
TABLE II

ONLINE BANG-BANG CHARGING COMPUTATIONAL RESULTS

\begin{tabular}{|c|c|c|c|c|c|c|c|}
\hline Networks & Profiles & Binary variables & $\mu$ & $\lambda$ & Obj. (9) by MICP (15) & Obj. (9) by snapshot (16) & Avg. Time (s) \\
\hline \multirow{4}{*}{ Case9 } & Profile 1 & 1512 & 1 & 1 & 54174.2 & 54193.6 & 11.3 \\
\hline & Profile 2 & 1512 & 1 & 1 & 54223.1 & 54276.8 & 11.5 \\
\hline & Profile 3 & 1512 & 1 & 1 & 54481.0 & 54485.2 & 11.7 \\
\hline & Profile 4 & 1512 & 1 & 1 & 54413.9 & 54427.7 & 11.8 \\
\hline \multirow{4}{*}{ Case14 } & Profile 1 & 2520 & 1 & 1 & 77174.8 & 77176.2 & 13.7 \\
\hline & Profile 2 & 2520 & 1 & 1 & 77224.9 & 77242.5 & 13.9 \\
\hline & Profile 3 & 2520 & 1 & 1 & 77760.3 & 77761.2 & 10.6 \\
\hline & Profile 4 & 2520 & 1 & 1 & 77394.4 & 77396.3 & 12.3 \\
\hline \multirow{4}{*}{ Case 30} & Profile 1 & 3012 & 10 & 1 & 6560.7 & 6560.8 & 24.0 \\
\hline & Profile 2 & 3012 & 10 & 1 & 6569.6 & 6569.7 & 23.1 \\
\hline & Profile 3 & 3012 & 10 & 1 & 6615.1 & 6615.2 & 25.2 \\
\hline & Profile 4 & 3012 & 10 & 1 & 6589.3 & 6599.1 & 29.4 \\
\hline \multirow{4}{*}{ Case 57} & Profile 1 & 3528 & 100 & 10 & 394104.8 & 394109.1 & 61.5 \\
\hline & Profile 2 & 3528 & 100 & 10 & 394667.8 & 394680.5 & 54.4 \\
\hline & Profile 3 & 3528 & 100 & 10 & 397493.8 & 397500.9 & 62.6 \\
\hline & Profile 4 & 3528 & 100 & 10 & 396552.6 & 396564.6 & 58.3 \\
\hline
\end{tabular}
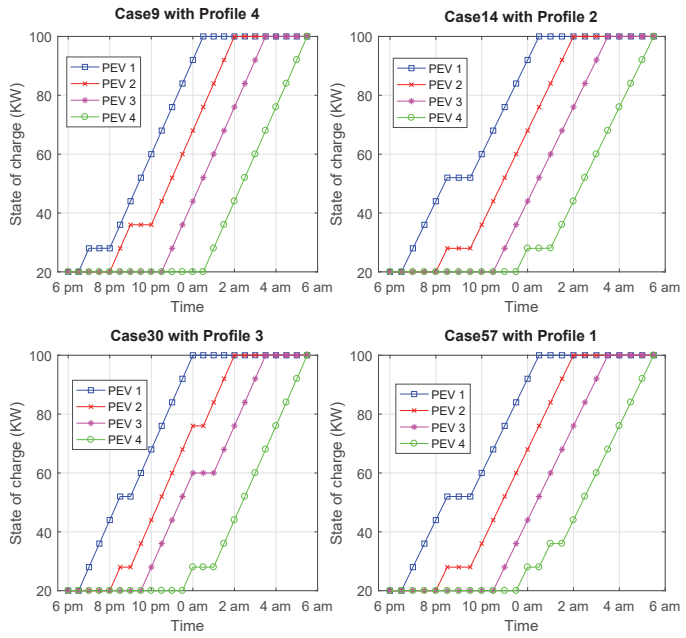

Fig. 3. The SOC of PEVs during the charging period

[6] J. F. Franco, M. J. Rider, and R. Romero, A mixed-integer linear programming model for the electric vehicle charging coordination problem in unbalanced electrical distribution systems, IEEE Trans. Smart Grid, vol. 6, no. 5, pp. 2200-2210, 2015.

[7] C. S. Antnez, J. F. Franco, M. J. Rider, R. Romero, A new methodology for the optimal charging coordination of electric vehicles considering vehicle-to-grid technology, IEEE Trans. Sustainable Energy, vol. 7, pp. 596-607, April 2016.

[8] K. Clement-Nyns, E. Haesen, J. Driesen, The impact of vehicle-togrid on the distribution grid, Electric Power Systems Research, vol. 81, no. 1, pp. 185-192, 2011.

[9] W. Tang and Y. J. A. Zhang, A model predictive control approach for low-complexity electric vehicle charging scheduling: optimality and scalability, IEEE Trans. Power Systems, vol. 32, no. 2, pp. 1050-1063, 2017.

[10] P. Malysz, S. Sirouspour, A. Emadi, An optimal energy storage control strategy for grid-connected microgrids, IEEE Trans. Smart Grid, vol. 5, pp. 1785-1796, 2014.

[11] A. Ravichandran, S. Sirouspour, P. Malysz, A. Emadi, "A chanceconstraints-based control strategy for microgrids with energy storage and integrated electric vehicles, IEEE Trans. Smart Grid, 2016.

[12] A. H. Phan, H. D. Tuan, H. H. Kha, D. T. Ngo, Nonsmooth optimization for efficient beamforming in cognitive radio multicast transmission, IEEE Trans. Sign. Process., vol. 60, no. 6, pp. 29412951, 2012

[13] Y. Shi, H. D. Tuan, H. Tuy, S. Su, Global optimization for optimal power flow over transmission networks, J. Global Optimz. vol. 69, pp. 745-760, 2017.
[14] Y. Shi, H. D. Tuan, A. V. Savkin, T. Q. Duong, H. V. Poor, Model predictive control for smart grids with multiple electric-vehicle charging stations, IEEE Trans. Smart Grid, vol. 10, pp. 2127-2136, 2019.

[15] Y. Shi, H. D. Tuan, P. Apkarian, Nonconvex spectral optimization algorithms for reduced-order $H_{\infty}$ LPV-LFT controllers, Int. J. Non. Robust Control, vol. 27, pp. 4421-4442, 2017.

[16] H. Tuy, Convex Analysis and Global Optimization (second edition). Springer International Publishing AG, 2017.

[17] E. Che, H. D. Tuan, and H. H. Nguyen: Joint optimization of cooperative beamforming and relay assignment in multi-user wireless relay networks, IEEE Trans. Wirel. Commun., vol. 13, pp. 5481-5495, (2014)

[18] H. H. M. Tam, H. D. Tuan, D. T. Ngo, T. Q. Duong, H. V. Poor, Joint load balancing and interference management for small-cell heterogeneous networks with limited backhaul capacity, IEEE Trans. Wirel. Commun., vol. 16, pp. 872-884, (2017)

[19] Y. Shi, H. D. Tuan, S. W. Su, H. H. M. Tam, Nonsmooth optimization for optimal power flow over transmission networks, in 2015 IEEE Global Conf. Signal Info. Process. (GlobalSIP), pp. 1141-1144, 2015.

[20] A. A. Nasir, H. D. Tuan, D. T. Ngo, T. Q. Duong, H. V. Poor, Beamforming design for wireless information and power transfer systems: Receive power-splitting versus transmit time-switching, IEEE Trans. Commun., vol. 65, no. 2, pp. 876-889, 2017.

[21] J. Sturm, Using SeDuMi 1.02, a MATLAB toolbox for optimization over symmetric cones, Optimization Methods and Software, vol. 1112, pp. 625-653, 1999.

[22] M. Grant, S. Boyd, CVX: Matlab software for disciplined convex programming, version 2.1. http://cvxr.com/cvx.

[23] R. D. Zimmerman, C. E. Murillo-Sanchez, and R. J. Thomas, Matpower: Steady-state operations, planning, and analysis tools for power systems research and education, IEEE Trans. Power Systems, vol. 26, pp. 12-19, 2011.

[24] The residential demand of the UK. http://www.gridwatch.templar.co. uk/download.php.

[25] The electricity price of the uk. https://www.businesselectricityprices. org.uk/retail-versus-wholesale-prices/. 